\title{
ENGAJAMENTO NO TRABALHO E SUAS CONTRIBUIÇÕES PARA A PRODUTIVIDADE EM UMA EMPRESA DE VAREJO MINEIRA
}

Danielle Vinte De Andrade Veiga ${ }^{1}$

Eliana M M Torga ${ }^{2}$

\footnotetext{
1 PROGRAMA DE PÓS GRADUAÇÃO STRICTO SENSU EM ADMINISTRAÇÃO / FACULDADE / Centro Universitário UNA

${ }^{2}$ PROGRAMA DE PÓS GRADUAÇÃO STRICTO SENSU EM ADMINISTRAÇÃO / Faculdade / Centro Universitário UNA
} 


\section{ENGAJAMENTO NO TRABALHO E SUAS CONTRIBUIÇÕES PARA A PRODUTIVIDADE EM UMA EMPRESA DE VAREJO MINEIRA}

Resumo: Este estudo objetivou analisar o poder preditivo do engajamento no trabalho sobre a percepção de produtividade dos trabalhadores de uma empresa familiar, de pequeno porte do ramo varejista. Realizou-se um levantamento tipo survey, de natureza descritiva, com base no método de Modelagem de Equações Estruturais, com estimação por Mínimos Quadrados Parciais, PLS-SEM, com variáveis quantitativas. Com base nos estudos obtidos nesta pesquisa, e em pesquisas anteriores sobre esses construtos, tornou-se possível propor um modelo que objetiva contribuir com os estudos sobre a maneira como o engajamento no trabalho impacta na produtividade da organização. As relações entre os construtos se mostraram positivas e estatisticamente significativas, o que indica que as hipóteses foram suportadas. A contribuição deste estudo foi a de trazer, para a discussão, o tema do engajamento no trabalho em relação à produtividade, enriquecendo e atualizando estudos acerca deste tema.

Palavras-chave: Engajamento. Produtividade. Empresa pequeno Porte. Varejo

\section{INTRODUÇÃO}

As estruturas organizacionais focadas no controle dos empregados e na produtividade em larga escala cedem lugar a uma estrutura em que o capital humano é o foco estratégico, a fim de superarem metas e alcançarem competitividade, colocando o indivíduo no centro das atenções. Assim, as organizações buscam encontrar e manter empregados competentes, satisfeitos, comprometidos, motivados e engajados com o negócio.

As empresas têm se tornado bem-sucedidas ao adotar traços positivos, tais como a agilidade, a flexibilidade, a valorização das relações pessoais e a capacidade de adaptação como formato de seus modelos de gestão (WOOD JR.; CALDAS, 2007).

As organizações têm se esforçado em maximizar sua eficiência, buscando o comprometimento e a motivação de seus funcionários para um melhor desempenho (KOYUNCU et al., 2006). Em todo o mundo, líderes e gerentes reconhecem que um dos elementos vitais para a eficácia das organizações está no engajamento de seus funcionários (WELCH, 2011).

Melhorar a produtividade dos funcionários tem sido um dos objetivos mais importantes para as organizações. Isso porque os níveis mais altos de produtividade dos funcionários fornecem várias vantagens a uma organização e aos funcionários (SHARMA; SHARMA, 2014).

Além disso, empregados que são mais produtivos podem obter melhores salários, melhores condições de trabalho e oportunidades de emprego favoráveis. Além disso, maior produtividade tende a maximizar a vantagem competitiva por meio da redução de custos e da melhoria na alta qualidade do produto (BAILY et al., 2005; HILL et al., 2014; WRIGHT, 2004). Todos esses benefícios tornaram, digna de atenção, a produtividade dos funcionários. Portanto, olhar seus antecedentes é muito importante para garantir a sobrevivência organizacional e o sucesso a longo prazo.

Diante dos fatos, é objetivo geral deste estudo analisar o poder preditivo do engajamento no trabalho sobre a percepção de produtividade dos trabalhadores de uma empresa de varejo.

Na empresa estudada mais de 70\% dos colaboradores recebem mensalmente o piso da 
transporte diariamente e $50 \%$ do plano de saúde, porém os outros $50 \%$ é custo do empregado e, por essa razão a adesão é de menos de $20 \%$ do total de empregados. Eles não possuem nenhum tipo de comissionamento pelo trabalho de venda realizado, possuem em sua maioria o cargo de Atendente de Loja e quando toda a unidade bate a meta preestabelecida mensalmente há uma premiação de menos de $10 \%$ do valor do salário.

Esta pesquisa é uma oportunidade para contribuir, de forma mais abrangente e efetiva, para a ampliação e disseminação do conhecimento quanto aos conceitos que envolvem os termos engajamento no trabalho e produtividade, além das dimensões que compõem esse engajamento. Além disso, a autora deste trabalho é psicóloga e lida com fatores relacionados a ambos os temas, no seu cotidiano, na gestão da área de recursos humanos e na prestação de serviços de consultoria em gestão de pessoas.

\section{FUNDAMENTAÇÃO TEÓRICA}

\subsection{Engajamento no trabalho}

O engajamento dos empregados tem sido uma das principais prioridades nos negócios, para as organizações e seus executivos. Uma atenção considerável tem sido dada ao estudo do engajamento e suas relações positivas com as medidas de eficácia organizacional, como o desempenho no trabalho, a produtividade, a qualidade das atividades desempenhadas, a satisfação do cliente e o crescimento dos negócios e lucros (KATARIA; GARG; RASTOGI, 2012).

O quadro 1, a seguir apresenta as principais definições acerca do tema:

Quadro 1 - Definições de Engajamento no Trabalho

\begin{tabular}{l} 
Definição e comparação do termo \\
\hline Um desejo intríseco e incutido no empregado; uma paixão \\
pela excelência \\
São elementos cognitivos emocionais e comportamentais \\
que estão relacionados ao papel do desempenho do \\
empregado \\
É o compromisso e envolvimento de um colaborador em \\
seu trabalho, visando à melhoria do desempenho \\
organizacional \\
É o envolvimento, dependendo das percepções e avaliações \\
dos funcionários, sobre suas experiências de trabalho \\
Está relacionado a envolvimento, entusiasmo e a satisfação \\
dos indivíduos diante de seu trabalho \\
Trata-se da atitude em que o funcionário possui em \\
melhorar seu trabalho, em prol de melhorias na eficácia \\
organizacional. \\
São altos níveis de investimento pessoal, em termos físicos, \\
cognitivos e emocionais, em que eles dispendem energias \\
nas tarefas de um trabalho
\end{tabular}

É um estado mental caracterizado por três dimensões (o vigor, a dedicação e a absorção)

Fleming e Asplund (2007, p. 2)

Shuck; Wollard (2010); Saks (2008); Andrew e Sofian (2011).

Sundaray (2011)

McEwen (2011)

Harter et al. (2002)

Robinson et al. (2004)

Acosta, Salanova, Llorens (2013); Bakker \& Leiter (2010); Salanova et al. (2000); Schaufeli (2002); Hahm (2017); Kataria, Garg, Rastogi (2012); Oliveira e Rocha (2017); Macey e Schneider (2008).

Bakker e Demerouti (2007); Bakker et al. (2008); Schaufeli et al. (2002) 
São sentimentos de realização relacionados ao trabalho e persistentes no tempo, apresentando, assim, uma natureza motivacional e social

É um indicador geral de bem-estar, relacionado de forma positiva ao desempenho de cada trabalhador

É uma experiência prazerosa para os funcionários, que impulsiona a proatividade e a inovação no local de trabalho, e aumenta a capacidade dos funcionários de se adaptarem às mudanças no local de trabalho, em situações de emergência

Produzir tarefas com a melhor qualidade possível, na certeza de que podem levar a empresa adiante
Bakker e Demerouti (2007); Bakker et al. (2008); Llorens et al. (2007); Salanova et al. (2000); Salanova e Schaufeli (2009); Schaufeli et al. (2002)

Xanthopoulou et al. (2009)

Kataria, Garg, Rastogi (2012)

Kennedy e Daim (2010)

Fonte: Elaborado pela autora (2018).

Fleming e Asplund (2007, p. 2) descrevem que o engajamento no trabalho é incutir um desejo intrínseco e uma paixão pela excelência nos corações e mentes dos empregados. Outros pesquisadores viram o engajamento no trabalho como um construto que consiste em elementos cognitivos, emocionais e comportamentais, relacionados ao papel do desempenho do empregado (SHUCK; WOLLARD, 2010). Para Sundaray (2011), é o engajamento que reflete o compromisso e envolvimento de um colaborador em seu trabalho, visando à melhoria do desempenho organizacional.

O engajamento no trabalho está relacionado a um estado mental caracterizado por três dimensões (o vigor, a dedicação e a absorção), sendo um construto motivacional positivo, que implica sentimentos de realização relacionados ao trabalho, e persistentes no tempo, apresentando assim uma natureza motivacional e social (BAKKER; DEMEROUTI, 2007; BAKKER et al., 2008; LLORENS et al., 2007; SALANOVA; SCHAUFELI, 2009; SCHAUFELI et al., 2002).

É notório, na literatura, que a promoção do engajamento no trabalho modula os efeitos diretos dos recursos organizacionais sobre o bem-estar, a qualidade de vida, em geral, e o desempenho (LLORENS et al., 2007). Isso gera benefícios, não somente no nível individual, mas também no nível das organizações, de forma a trazer mais vantagem competitiva para elas (BAKKER et al., 2007; BAKKER; LEITER, 2010; SALANOVA; SCHAUFELI, 2000).

No Quadro 2, estão detalhadas as características das dimensões de engajamento no trabalho. A classificação das dimensões baseou-se em definições que sistematicamente são apontadas por autores em diversos estudos.

Quadro 2 - Dimensões de engajamento no trabalho

\begin{tabular}{|c|c|}
\hline DIMENSÕES & MEDIDOS NAS AFIRMAÇÕES \\
\hline & $\begin{array}{l}\text { 1- No meu trabalho, sinto que estou cheio de energia; } 4 \text { - No meu trabalho, sinto-me forte e } \\
\text { cheio de vigor; } 8 \text { - Tenho vontade de ir para o trabalho, quando levanto de manhã; } 12 \text { - Eu }\end{array}$ \\
\hline VIGOR & $\begin{array}{l}\text { posso me manter trabalhando por períodos de tempo muito longos; } 15 \text { - Eu consigo me adap- } \\
\text { tar mentalmente às situações difíceis no meu trabalho; } 17 \text { - Em relação ao meu trabalho, sou } \\
\text { persistente mesmo quando as coisas não dão certo. }\end{array}$ \\
\hline DEDICAÇÃO & $\begin{array}{l}\text { 2- Eu considero meu trabalho cheio de significado e propósito; 5- Sou entusiasmado com } \\
\text { meu trabalho; } 7 \text { - Meu trabalho me inspira; } 10 \text { - Tenho orgulho do trabalho que realizo; } 13 \text { - } \\
\text { Para mim, o meu trabalho é desafiador. }\end{array}$ \\
\hline ABSORÇÃO & 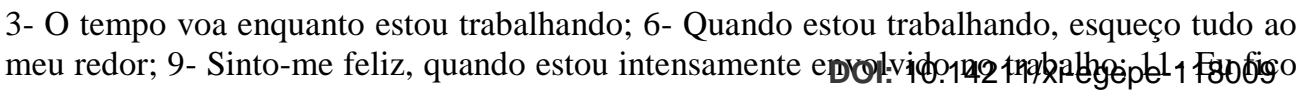 \\
\hline
\end{tabular}


Fonte: Adaptado pela autora (2018).

\subsubsection{A dimensão - Vigor}

Segundo Bakker e Demerouti (2007), o vigor pode ser descrito em termos dos níveis de energia de um funcionário e da resiliência mental enquanto faz o trabalho. Shirom (2003) indicou que vigor se refere à saúde mental e física de um empregado.

O componente vigor indica: altos níveis de energia positiva e resiliência mental, enquanto trabalha; vontade de investir tempo; e desejo de esforçar-se nas tarefas do trabalho caracterizadas por persistência (SCHAUFELI, 2002).

\subsubsection{A dimensão - Dedicação}

A dedicação está relacionada ao envolvimento do empregado. Ela tem relação com aspectos emocionais em que o empregado se encontra plenamente concentrado e envolvido em seu trabalho: um estado em que os empregados percebem uma razão para seu trabalho (ACOSTA, SALANOVA; LLORENS, 2013; BAKKER; LEITER, 2010; BAKKER et al., 2011; GONZÁLEZ-ROMÁ et al., 2006; SALANOVA et al., 2000; SCHAUFELI, 2002). Para Yongxing et al. (2017), a dedicação é um estado em que os funcionários encontram significado para seu trabalho.

\subsubsection{A dimensão - Absorção}

A outra dimensão do engajamento do trabalho, conhecida como absorção, foi previamente descrita por ser totalmente focada e, felizmente, ligada ao trabalho de alguém. É por meio dela que o funcionário sente que o tempo passa rapidamente e tem dificuldades em se separar trabalho (TRUSS et al., 2013).

Absorção refere-se ao aspecto cognitivo que os empregados experimentam quando estão trabalhando, de forma que o envolvimento e a concentração, por eles percebidos, são tão fortes que eles sentem dificuldades para se separarem do trabalho (BAKKER et al., 2008; GONZÁLEZ-ROMÁ et al., 2006; KATARIA; GARG; RASTOGI, 2012; SCHAUFELI et al., 2002; SALANOVA; SCHAUFELI, 2009; XANTHOPOULOU et al., 2009).

\subsection{Produtividade}

Para Singh, Motwani e Kumar (2000), a produtividade representa uma das variáveis mais importantes que regem a economia. $\mathrm{O}$ termo produtividade tem sido comumente utilizado e, ao longo das últimas décadas, inúmeros têm sido os estudiosos que buscam defini-lo. Para Martins e Laugeni (2006), a produtividade, que é compreendida como uma soma de atividades passíveis de levar à transformação de um bem tangível em outro, de maior utilidade, acompanha o homem desde os primórdios.

O quadro 3, a seguir, apresenta algumas definições do tema: 


\begin{tabular}{|c|c|}
\hline Definições & Referências \\
\hline Capacidade de produzir & $($ LITTRÉ, 1883) \\
\hline É aquilo que o homem realiza com o material, com o capital e a tecnolo- & (JAPAN \\
\hline gia. A produtividade é uma questão pessoal. Depende de uma atitude que & PRODUCTIVITY \\
\hline devemos ter para melhorar continuamente a nós mesmos e aquilo que está & CENTRE, 1958, apud \\
\hline Produtividade $=$ unidades de outputs/unidades de inputs & (CHEW, 1988) \\
\hline Produtividade $=$ actual output/recursos utilizados esperados & (SINK; TUTTLE, 1989) \\
\hline Produtividade $=$ receita total $($ custo + objetivo de lucro $)$ & (FISHER, 1990) \\
\hline Produtividade $=$ valor adicionado/input de fatores de produção & (ASPÉN et al., 1991) \\
\hline $\begin{array}{l}\text { A produtividade é definida como a relação do que é produzido com o que } \\
\text { é necessário para produzi-lo. A produtividade mede a relação entre output } \\
\text { - tais como bens e serviços produzidos e input - que inclui o trabalho } \\
\text { capital materiais e outros recursos }\end{array}$ & (HILL, 1993) \\
\hline $\begin{array}{l}\text { Produtividade (output por hora de trabalho) é o fator central de longo pra- } \\
\text { zo, determinando qualquer média de vida da população }\end{array}$ & (THUROW, 1993) \\
\hline $\begin{array}{l}\text { Produtividade = a qualidade ou estado de levar em diante, de gerar de } \\
\text { causar a existência, de produzir resultados grandes ou produzir abundan- } \\
\text { temente }\end{array}$ & (KOSS; LEWIS, 1993) \\
\hline $\begin{array}{l}\text { Produtividade significa o quanto e como se produz a partir dos recursos } \\
\text { utilizados. Se produzirmos mais ou melhores bens a partir dos mesmos } \\
\text { recursos, aumentamos a produtividade. Ou se produzimos os mesmos bens } \\
\text { com menores recursos, também aumentamos a produtividade. Por "recur- } \\
\text { sos", entendemos todos os recursos humanos e físicos, isto é, as pessoas } \\
\text { que produzem os bens ou fornecem os serviços e os bens com que as pes- } \\
\text { soas podem produzi-los ou prestar os serviços. }\end{array}$ & (BERNOLAK, 1997) \\
\hline $\begin{array}{l}\text { Produtividade é a comparação dos inputs físicos a uma fábrica com os } \\
\text { outputs físicos da fábrica }\end{array}$ & $\begin{array}{l}\text { (KAPLAN; COOPER, } \\
\text { 1998) }\end{array}$ \\
\hline $\begin{array}{l}\text { Produtividade }=\text { eficiência } * \text { eficácia }=\text { valor adicionando tempo/tempo } \\
\text { total }\end{array}$ & $\begin{array}{l}\text { (JACKSON; PETERS- } \\
\text { SON, 1999) }\end{array}$ \\
\hline $\begin{array}{l}\text { Produtividade }=(\text { output/input }) * \text { qualidade }=\text { eficiência } * \text { utilização } * \text { qua- } \\
\text { lidade }\end{array}$ & (AL-DARRAB, 2000) \\
\hline $\begin{array}{l}\text { Produtividade é a capacidade de satisfazer as necessidades do mercado de } \\
\text { bens e serviços com um mínimo de consumo de recursos }\end{array}$ & $\begin{array}{l}\text { (MOSENG; ROLSTA- } \\
\text { DAS, 2001) }\end{array}$ \\
\hline
\end{tabular}

Fonte: Tangen (2005, p. 36).

A produtividade pode ser entendida como uma medida dos resultados em relação aos objetivos, ou seja, como a quantidade de serviços ou produtos gerados por um sistema (PRITCHARD, 1992).

São diversos os indicadores por meio dos quais é possível mensurar a produtividade: as vendas, o retorno do investimento, a quota do mercado, dentre outros (CUNHA et al., 2003). Mas, para esta pesquisa, torna-se relevante o indicador output empregado, que é o produto que advém do trabalho deste.

Em termos reais, a produtividade é um componente que afeta diretamente os lucros da empresa (GUMMESSON, 1998; SELS et al., 2006). Ela pode ser avaliada em termos da produção de um empregado, em um período específico. Normalmente, a produtividade de um determinado trabalhador será avaliada em relação a uma média de tempo, gasto por outros funcionários, que fazem um trabalho semelhante (OBDULIO, 2014).

Para essa pesquisa, o foco da produtividade está relacionado ao produto entregue pelo trabalhador. São elementos advindos de questões comportamentais, tanto individuais como da própria organização, sendo o entendimento de produtividade, trazido nessa pesquisa, aquele voltado para a concepção de produtividade tal como definida pela psicologia (CUNHA, $2003)$

DOI: 10.14211/xi-egepe-118009 


\section{METODOLOGIA}

Quanto aos fins, a presente pesquisa classifica-se como descritiva, pois busca compreender a relação do engajamento na produtividade. E tem o objetivo de descrever características de uma determinada amostra, por meio de técnicas, métodos, modelos e de uma fundamentada teoria para a realização da mesma (RAUPP, 2006).

Optou-se realizar uma pesquisa quantitativa, que se baseia em registros e análises de todos os dados numéricos, que refletem a percepção dos trabalhadores acerca do tema engajamento, estruturado em um questionário. (MINAYO, 2002)

Quantos aos meios, essa pesquisa constitui um survey, pois visa, por meio de um instrumento de pesquisa (LITWIN; FINK, 1995), obter dados ou informações sobre características, ações ou opiniões de um determinado grupo de pessoas, ao qual será feita a aplicação de questionários, que são a "técnica mais conhecida para se trabalhar neste sentido" (DALFOVO; LANA; SILVEIRA, 2008). Foi desenvolvido por meio de um estudo transversal único, ou seja, envolve a coleta de informações da amostra de elementos da população somente uma vez.

Para isso, foi utilizado um questionário com uma escala tipo Likert, de 5 pontos: Discordo totalmente (1) Discordo (2) Nem discordo nem concordo (3) Concordo (4) Concordo totalmente (5), por meio de escalas validadas em pesquisas realizadas com os mesmos construtos.

A pesquisa foi realizada com empregados de uma empresa de pequeno porte do ramo de varejo que possui, atualmente, 305 funcionários, distribuídos em suas dezesseis unidades de negócio, a serem selecionadas por conveniência, nas cidades de Belo Horizonte, Contagem, Betim, Vespasiano, Santa Luzia, Divinópolis, Nova Serrana, Sete Lagoas, Lagoa Santa, Conselheiro Lafaiete e Pedro Leopoldo, todas em Minas Gerais.

O tratamento de dados para análise dos resultados é a análise estatística multivariada, utilizando a modelagem de equações estruturais (HAIR et al., 2017). A técnica escolhida para análise dos dados é a Modelagem de Equações Estruturais, com estimação por Mínimos Quadrados Parciais (PLS-SEM), em função dos seguintes motivos: existência de múltiplos relacionamentos entre as variáveis dependentes e independentes, dados não normais, tamanho da amostra e contraste de predição posterior ao problema de pesquisa. Essa técnica de segunda geração, bastante útil nas pesquisas sociais, envolve diversas atividades e decisões do pesquisador.

\section{ANÁLISE DOS RESULTADOS}

Inicialmente, analisou-se média e o desvio padrão por grupos de homens e mulheres, para verificar a diferença na percepção dos gêneros, conforme tabela 1, a seguir:

Tabela 1 - Estatísticas de grupo: média e o desvio padrão por grupos de homens e mulheres.

\begin{tabular}{|c|c|c|c|c|c|}
\hline Enunciados & Sexo & $\mathbf{N}$ & Média & $\begin{array}{c}\text { Erro } \\
\text { Desvio }\end{array}$ & $\begin{array}{c}\text { Erro } \\
\text { padrão } \\
\text { da média }\end{array}$ \\
\hline \multirow{2}{*}{ Sou requisitado para implementar tarefas novas. } & Homem & 94 & 3,9362 & 0,9318 & 0,0014 \\
\hline & Mulher & 134 & 3,8134 & 0,9074 & 0,0011 \\
\hline \multirow{2}{*}{ Consigo concluir as tarefas planejadas. } & Homem & 94 & 4,1596 & 0,6889 & 0,0010 \\
\hline & Mulher & 134 & 4,0448 & 0,6677 & 0,0008 \\
\hline Me lemhrn de recnnnder uma colicitarãn e nu & Homem & 94 & DQ) $\mid 9(1 \Phi 31$ & $1(1 \times x)$ & $-1+80092$ \\
\hline
\end{tabular}




\begin{tabular}{|c|c|c|c|c|c|}
\hline Enunciados & Sexo & $\mathbf{N}$ & Média & $\begin{array}{c}\text { Erro } \\
\text { Desvio }\end{array}$ & $\begin{array}{c}\text { Erro } \\
\text { padrão } \\
\text { da média }\end{array}$ \\
\hline \multirow{2}{*}{$\begin{array}{l}\text { É fácil concentrar nas tarefas sobre minha } \\
\text { responsabilidade. }\end{array}$} & Homem & 94 & 3,9255 & 0,7472 & 0,0011 \\
\hline & Mulher & 134 & 3,9478 & 0,7362 & 0,0009 \\
\hline \multirow{2}{*}{ Costumo sugerir ideias novas no trabalho. } & Homem & 94 & 4,0745 & 0,8535 & 0,0012 \\
\hline & Mulher & 134 & 3,9104 & 0,7961 & 0,0010 \\
\hline \multirow{2}{*}{$\begin{array}{l}\text { Trabalho mais que o esperado para completar } \\
\text { minhas tarefas. }\end{array}$} & Homem & 94 & 3,2660 & 0,9912 & 0,0014 \\
\hline & Mulher & 134 & 3,2313 & 1,0574 & 0,0013 \\
\hline \multirow{2}{*}{ No trabalho sinto-me cheio de energia. } & Homem & 94 & 3,8511 & 1,0310 & 0,0015 \\
\hline & Mulher & 134 & 3,7015 & 0,8812 & 0,0011 \\
\hline \multirow{2}{*}{ No trabalho tenho força e vitalidade. } & Homem & 94 & 3,9043 & 0,8638 & 0,0013 \\
\hline & Mulher & 134 & 3,7612 & 0,7934 & 0,0010 \\
\hline \multirow{2}{*}{$\begin{array}{l}\text { Quando levanto pela manhã tenho vontade de ir } \\
\text { trabalhar. }\end{array}$} & Homem & 94 & 3,7766 & 1,0734 & 0,0016 \\
\hline & Mulher & 134 & 3,8433 & 0,9212 & 0,0011 \\
\hline \multirow{2}{*}{$\begin{array}{l}\text { Posso continuar trabalhando durante longos } \\
\text { períodos de tempo. }\end{array}$} & Homem & 94 & 3,7766 & 1,0635 & 0,0016 \\
\hline & Mulher & 134 & 3,6119 & 0,9843 & 0,0012 \\
\hline \multirow{2}{*}{ Sou mentalmente flexível no trabalho. } & Homem & 94 & 4,1596 & 0,7191 & 0,0010 \\
\hline & Mulher & 134 & 4,0597 & 0,6201 & 0,0008 \\
\hline \multirow{2}{*}{$\begin{array}{l}\text { Sou persistente mesmo quando as coisas não vão } \\
\text { bem. }\end{array}$} & Homem & 94 & 4,1489 & 0,7713 & 0,0011 \\
\hline & Mulher & 134 & 4,1045 & 0,7848 & 0,0010 \\
\hline \multirow{2}{*}{$\begin{array}{l}\text { O trabalho que realizo é cheio de significado e } \\
\text { propósito. }\end{array}$} & Homem & 94 & 4,0745 & 0,9020 & 0,0013 \\
\hline & Mulher & 134 & 4,0672 & 0,6824 & 0,0008 \\
\hline \multirow{2}{*}{ Estou entusiasmado com meu trabalho. } & Homem & 94 & 3,8511 & 1,0715 & 0,0016 \\
\hline & Mulher & 134 & 3,8358 & 0,8826 & 0,0011 \\
\hline \multirow{2}{*}{ Meu trabalho me inspira. } & Homem & 94 & 3,7872 & 0,9986 & 0,0015 \\
\hline & Mulher & 134 & 3,8060 & 0,9017 & 0,0011 \\
\hline \multirow{2}{*}{ Estou orgulhoso com o trabalho que realizo. } & Homem & 94 & 4,0851 & 0,8336 & 0,0012 \\
\hline & Mulher & 134 & 4,0373 & 0,8500 & 0,0010 \\
\hline \multirow{2}{*}{ Meu trabalho é desafiador. } & Homem & 94 & 4,0957 & 0,8881 & 0,0013 \\
\hline & Mulher & 134 & 3,8507 & 0,8853 & 0,0011 \\
\hline \multirow{2}{*}{ Mantenho-me envolvido com trabalho que realizo. } & Homem & 94 & 4,2021 & 0,6617 & 0,0010 \\
\hline & Mulher & 134 & 4,2090 & 0,6473 & 0,0008 \\
\hline \multirow{2}{*}{ O "tempo voa" quando estou trabalhando. } & Homem & 94 & 3,9043 & 1,0626 & 0,0015 \\
\hline & Mulher & 134 & 3,7388 & 0,9137 & 0,0011 \\
\hline \multirow{2}{*}{$\begin{array}{l}\text { Esqueço tudo o que se passa ao meu redor quando } \\
\text { estou trabalhando. }\end{array}$} & Homem & 94 & 3,1702 & 1,1170 & 0,0016 \\
\hline & Mulher & 134 & 3,2761 & 0,9494 & 0,0012 \\
\hline \multirow{2}{*}{ Sinto-me feliz quando trabalho intensamente. } & Homem & 94 & 3,7872 & 1,0092 & 0,0015 \\
\hline & Mulher & 134 & 3,8060 & 0,8765 & 0,0011 \\
\hline \multirow{2}{*}{ Sinto-me envolvido com o trabalho que faço. } & Homem & 94 & 4,0851 & 0,9186 & 0,0013 \\
\hline & Mulher & 134 & 4,0373 & 0,7061 & 0,0009 \\
\hline \multirow{2}{*}{ Deixo-me levar pelo meu trabalho. } & Homem & 94 & 3,7128 & 0,8458 & 0,0012 \\
\hline & Mulher & 134 & 3,7239 & 0,7954 & 0,0010 \\
\hline \multirow{2}{*}{ É difícil desligar-me do trabalho. } & Homem & 94 & 3,2234 & 1,1864 & 0,0017 \\
\hline & Mulher & 134 & 3,3358 & 1,0290 & 0,0013 \\
\hline
\end{tabular}


Ainda se realizou a comparação de amostras independentes por meio do test-t, conforme tabela 2, a seguir:

Tabela 2 - Test- $t$ de amostras independentes

\begin{tabular}{|c|c|c|c|c|c|c|c|}
\hline Enunciados & $\begin{array}{l}\text { Igualdade } \\
\text { de Médias }\end{array}$ & $\mathbf{t}$ & $\begin{array}{c}\text { Nível de } \\
\text { Significânc } \\
\text { ia } \\
\end{array}$ & p & $\begin{array}{l}\text { Diferença } \\
\text { média }\end{array}$ & $\begin{array}{c}\text { Erro } \\
\text { padrão da } \\
\text { diferença } \\
\end{array}$ & 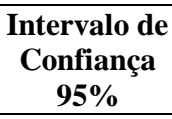 \\
\hline \multirow{2}{*}{$\begin{array}{l}\text { Sou requisitado para } \\
\text { implementar tarefas } \\
\text { novas. }\end{array}$} & VIA & 70,3111 & $* * *$ & 0,0000 & 0,1227 & 0,0017 & $\begin{array}{c}0,1193 ; \\
0,1262]\end{array}$ \\
\hline & VINA & 69,9847 & $* * *$ & 0,0000 & 0,1227 & 0,0018 & $\begin{array}{c}{[0,1193} \\
0,1262]\end{array}$ \\
\hline \multirow{2}{*}{$\begin{array}{l}\text { Consigo concluir as } \\
\text { tarefas planejadas. }\end{array}$} & VIA & 89,1989 & $* * *$ & 0,0000 & 0,1148 & 0,0013 & $\begin{array}{c}{[0,1123} \\
0,1173]\end{array}$ \\
\hline & VINA & 88,7108 & $* * *$ & 0,0000 & 0,1148 & 0,0013 & $\begin{array}{c}{[0,1123 ;} \\
0,1173]\end{array}$ \\
\hline \multirow{2}{*}{$\begin{array}{l}\text { Me lembro de responder } \\
\text { uma solicitação e ou } \\
\text { pedido. }\end{array}$} & VIA & $-81,5159$ & $* * *$ & 0,0000 & $-0,1107$ & 0,0014 & $\begin{array}{l}{[-0,1133 ;-} \\
0,1080]\end{array}$ \\
\hline & VINA & $-77,6712$ & $* * *$ & 0,0000 & $-0,1107$ & 0,0014 & $\begin{array}{c}{[-0,1135 ;-} \\
0,1079]\end{array}$ \\
\hline \multirow{2}{*}{$\begin{array}{l}\text { É fácil concentrar nas } \\
\text { tarefas sobre minha } \\
\text { responsabilidade. }\end{array}$} & VIA & $-15,7732$ & $* * *$ & 0,0000 & $-0,0222$ & 0,0014 & $\begin{array}{l}{[-0,0250 ;-} \\
0,0195]\end{array}$ \\
\hline & VINA & $-15,7324$ & $* * *$ & 0,0000 & $-0,0222$ & 0,0014 & $\begin{array}{l}{[-0,0250 ;-} \\
0,0195]\end{array}$ \\
\hline \multirow{2}{*}{$\begin{array}{l}\text { Costumo sugerir ideias } \\
\text { novas no trabalho. }\end{array}$} & VIA & 105,1050 & $* * *$ & 0,0000 & 0,1640 & 0,0016 & $\begin{array}{c}{[0,1610} \\
0,1671]\end{array}$ \\
\hline & VINA & 103,8306 & $* * *$ & 0,0000 & 0,1640 & 0,0016 & $\begin{array}{c}{[0,1609} \\
0,1671]\end{array}$ \\
\hline \multirow{2}{*}{$\begin{array}{l}\text { Trabalho mais que o } \\
\text { esperado para completar } \\
\text { minhas tarefas. }\end{array}$} & VIA & 17,6534 & $* * *$ & 0,0000 & 0,0346 & 0,0020 & $\begin{array}{l}{[0,0308} \\
0,0385]\end{array}$ \\
\hline & VINA & 17,8546 & $* * *$ & 0,0000 & 0,0346 & 0,0019 & $\begin{array}{c}{[0,0308 ;} \\
0,0384]\end{array}$ \\
\hline \multirow{2}{*}{$\begin{array}{l}\text { No trabalho sinto- } \\
\text { me cheio de energia. }\end{array}$} & VIA & 83,1198 & $* * *$ & 0,0000 & 0,1496 & 0,0018 & $\begin{array}{c}{[0,1460} \\
0,1531]\end{array}$ \\
\hline & VINA & 80,8799 & $* * *$ & 0,0000 & 0,1496 & 0,0018 & $\begin{array}{c}{[0,1459} \\
0,1532]\end{array}$ \\
\hline \multirow{2}{*}{$\begin{array}{l}\text { No trabalho tenho força } \\
\text { e vitalidade. }\end{array}$} & VIA & 91,3497 & $* * *$ & 0,0000 & 0,1431 & 0,0016 & $\begin{array}{c}{[0,1400 ;} \\
0,1461]\end{array}$ \\
\hline & VINA & 90,0012 & $* * *$ & 0,0000 & 0,1431 & 0,0016 & $\begin{array}{c}{[0,1399} \\
0,1462]\end{array}$ \\
\hline \multirow{2}{*}{$\begin{array}{l}\text { Quando levanto pela } \\
\text { manhã tenho vontade de } \\
\text { ir trabalhar. }\end{array}$} & VIA & $-35,5220$ & $* * *$ & 0,0000 & $-0,0667$ & 0,0019 & $\begin{array}{l}{[-0,0704 ;-} \\
0,0630]\end{array}$ \\
\hline & VINA & $-34,5885$ & $* * *$ & 0,0000 & $-0,0667$ & 0,0019 & $\begin{array}{l}{[-0,0705 ;-} \\
0,0629]\end{array}$ \\
\hline \multirow{2}{*}{$\begin{array}{l}\text { Posso } \\
\text { trabalhando continuar } \\
\text { longos períodos durante } \\
\text { tempo. }\end{array}$} & VIA & 85,0443 & $* * *$ & 0,0000 & 0,1647 & 0,0019 & $\begin{array}{c}{[0,1609} \\
0,1685]\end{array}$ \\
\hline & VINA & 83,9000 & $* * *$ & 0,0000 & 0,1647 & 0,0020 & $\begin{array}{c}{[0,1608} \\
0,1685]\end{array}$ \\
\hline \multirow{2}{*}{$\begin{array}{l}\text { Sou mentalmente } \\
\text { flexível no trabalho. }\end{array}$} & VIA & 79,2160 & $* * *$ & 0,0000 & 0,0999 & 0,0013 & $\begin{array}{c}{[0,0974} \\
0,1023]\end{array}$ \\
\hline & VINA & 77,1978 & $* * *$ & 0,0000 & 0,0999 & 0,0013 & $\begin{array}{c}{[0,0973} \\
0,1024]\end{array}$ \\
\hline \multirow{2}{*}{$\begin{array}{l}\text { Sou persistente mesmo } \\
\text { quando as coisas não } \\
\text { vão bem. }\end{array}$} & VIA & 29,9882 & $* * *$ & 0,0000 & 0,0445 & 0,0015 & $\begin{array}{c}{[0,0416} \\
0,0474]\end{array}$ \\
\hline & VINA & 30,0800 & $* * *$ & 0,0000 & 0,0445 & 0,0015 & $\begin{array}{c}{[0,0416} \\
0,0474]\end{array}$ \\
\hline O trabalho que realizo é & VIA & 4.9191 & $* * *$ & 0.0000 & 0.00013 & $4200 / x$ & epe-10.840.99 \\
\hline
\end{tabular}




\begin{tabular}{|c|c|c|c|c|c|c|c|}
\hline Enunciados & $\begin{array}{l}\text { Igualdade } \\
\text { de Médias }\end{array}$ & $\mathbf{t}$ & $\begin{array}{c}\text { Nível de } \\
\text { Significânc } \\
\text { ia } \\
\end{array}$ & $\mathbf{p}$ & $\begin{array}{l}\text { Diferença } \\
\text { média }\end{array}$ & $\begin{array}{c}\text { Erro } \\
\text { padrão da } \\
\text { diferença } \\
\end{array}$ & $\begin{array}{c}\text { Intervalo de } \\
\text { Confiança } \\
95 \% \\
\end{array}$ \\
\hline propósito. & VINA & 4,6897 & $* * *$ & 0,0000 & 0,0073 & 0,0016 & $\begin{array}{c}0,0043 ; \\
0,0104]\end{array}$ \\
\hline \multirow{2}{*}{$\begin{array}{l}\text { Estou entusiasmado com } \\
\text { meu trabalho. }\end{array}$} & VIA & 8,3030 & $* * *$ & 0,0000 & 0,0152 & 0,0018 & $\begin{array}{c}{[0,0116 ;} \\
0,0188]\end{array}$ \\
\hline & VINA & 8,0286 & $* * *$ & 0,0000 & 0,0152 & 0,0019 & $\begin{array}{c}{[0,0115 ;} \\
0,0190]\end{array}$ \\
\hline \multirow{2}{*}{ Meu trabalho me inspira. } & VIA & $-10,4447$ & $* * *$ & 0,0000 & $-0,0187$ & 0,0018 & $\begin{array}{c}{[-0,0223 ;-} \\
0,0152]\end{array}$ \\
\hline & VINA & $-10,2599$ & $* * *$ & 0,0000 & $-0,0187$ & 0,0018 & $\begin{array}{c}{[-0,0223 ;-} \\
0,0152]\end{array}$ \\
\hline \multirow{2}{*}{$\begin{array}{l}\text { Estou orgulhoso com o } \\
\text { trabalho que realizo. }\end{array}$} & VIA & 29,7902 & $* * *$ & 0,0000 & 0,0478 & 0,0016 & $\begin{array}{c}{[0,0446 ;} \\
0,0509]\end{array}$ \\
\hline & VINA & 29,8921 & $* * *$ & 0,0000 & 0,0478 & 0,0016 & $\begin{array}{c}{[0,0447 ;} \\
0,0509]\end{array}$ \\
\hline \multirow{2}{*}{$\begin{array}{l}\text { Meu trabalho } \\
\text { desafiador. }\end{array}$} & VIA & 145,2765 & $* * *$ & 0,0000 & 0,2450 & 0,0017 & $\begin{array}{c}{[0,2417} \\
0,2483]\end{array}$ \\
\hline & VINA & 145,1953 & $* * *$ & 0,0000 & 0,2450 & 0,0017 & $\begin{array}{c}{[0,2417} \\
0,2483]\end{array}$ \\
\hline \multirow{2}{*}{$\begin{array}{l}\text { Mantenho-me } \\
\text { envolvido com trabalho } \\
\text { que realizo. }\end{array}$} & VIA & $-5,4933$ & $* * *$ & 0,0000 & $-0,0068$ & 0,0012 & $\begin{array}{c}{[-0,0093 ;-} \\
0,0044]\end{array}$ \\
\hline & VINA & $-5,4722$ & $* * *$ & 0,0000 & $-0,0068$ & 0,0012 & $\begin{array}{c}{[-0,0093 ;-} \\
0,0044]\end{array}$ \\
\hline \multirow{2}{*}{$\begin{array}{l}\text { O "tempo voa" quando } \\
\text { estou trabalhando. }\end{array}$} & VIA & 88,9374 & $* * *$ & 0,0000 & 0,1654 & 0,0019 & $\begin{array}{c}{[0,1618} \\
0,1691]\end{array}$ \\
\hline & VINA & 86,6287 & $* * *$ & 0,0000 & 0,1654 & 0,0019 & $\begin{array}{c}{[0,1617 ;} \\
0,1692]\end{array}$ \\
\hline \multirow{2}{*}{$\begin{array}{l}\text { Esqueço tudo o que se } \\
\text { passa ao meu redor } \\
\text { quando } \\
\text { trabalhando. }\end{array}$} & VIA & $-54,4784$ & $* * *$ & 0,0000 & $-0,1059$ & 0,0019 & $\begin{array}{c}{[-0,1097 ;-} \\
0,1021]\end{array}$ \\
\hline & VINA & $-52,9596$ & $* * *$ & 0,0000 & $-0,1059$ & 0,0020 & $\begin{array}{c}{[-0,1098 ;-} \\
0,1020]\end{array}$ \\
\hline \multirow{2}{*}{$\begin{array}{l}\text { Sinto-me feliz quando } \\
\text { trabalho intensamente. }\end{array}$} & VIA & $-10,5493$ & $* * *$ & 0,0000 & $-0,0187$ & 0,0018 & $\begin{array}{c}{[-0,0222 ;-} \\
0,0153]\end{array}$ \\
\hline & VINA & $-10,2933$ & $* * *$ & 0,0000 & $-0,0187$ & 0,0018 & $\begin{array}{c}{[-0,0223 ;-} \\
0,0152]\end{array}$ \\
\hline \multirow{2}{*}{$\begin{array}{l}\text { Sinto-me envolvido com } \\
\text { o trabalho que faço. }\end{array}$} & VIA & 31,3787 & $* * *$ & 0,0000 & 0,0478 & 0,0015 & $\begin{array}{c}{[0,0448 ;} \\
0,0508]\end{array}$ \\
\hline & VINA & 29,9936 & $* * *$ & 0,0000 & 0,0478 & 0,0016 & $\begin{array}{c}{[0,0447 ;} \\
0,0509]\end{array}$ \\
\hline \multirow{2}{*}{$\begin{array}{l}\text { Deixo-me levar pelo } \\
\text { meu trabalho. }\end{array}$} & VIA & $-7,1545$ & $* * *$ & 0,0000 & $-0,0111$ & 0,0016 & $\begin{array}{l}{[-0,0142 ;-} \\
0,0081]\end{array}$ \\
\hline & VINA & $-7,0779$ & $* * *$ & 0,0000 & $-0,0111$ & 0,0016 & $\begin{array}{c}{[-0,0142 ;-} \\
0,0080]\end{array}$ \\
\hline \multirow{2}{*}{$\begin{array}{l}\text { É difícil desligar-me do } \\
\text { trabalho. }\end{array}$} & VIA & $-53,8820$ & $* * *$ & 0,0000 & $-0,1124$ & 0,0021 & $\begin{array}{c}{[-0,1165 ;-} \\
0,1083]\end{array}$ \\
\hline & VINA & $-52,5618$ & $* * *$ & 0,0000 & $-0,1124$ & 0,0021 & $\begin{array}{c}{[-0,1166 ;-} \\
0,1082]\end{array}$ \\
\hline
\end{tabular}

Nota: VIA = Variâncias iguais assumidas. VINA = Variâncias iguais não assumidas. $* * * p<0,01$. Fonte: Dados da pesquisa.

Posteriormente, tomando por base as cargas fatoriais constata-se que as dependências foram relacionadas positivamente, de forma que o construto produtividade obteve um valor de $\mathrm{R}^{2}=0,2695$. Isso indica que o engajamento no trabalho, com seus antecedentes, é capaz de explicar a variação de aproximadamente $27 \%$ da produtividade dهotabalhłallorxi-egepe-118009 
De modo análogo, ao segmentar os dados por grupo de homens e mulheres, obtêm-se, os valores de $\mathrm{R}^{2}=0,2904$ e $\mathrm{R}^{2}=0,3229$, respectivamente. Isso significa que a produtividade para os homens equivale a cerca de $29 \%$ e, para as mulheres, a cerca de $32 \%$. Há uma mensuração equivalente total e isso indica que é possível executar a análise multigrupo. Ao comparar homens e mulheres, verifica-se, que há diferença estatisticamente significante apenas na relação entre absorção e engajamento no trabalho.

As sínteses dos resultados sobre o modelo da pesquisa são apresentadas na

Figura 1 - Síntese dos Resultados

1.

Figura 1 - Síntese dos Resultados

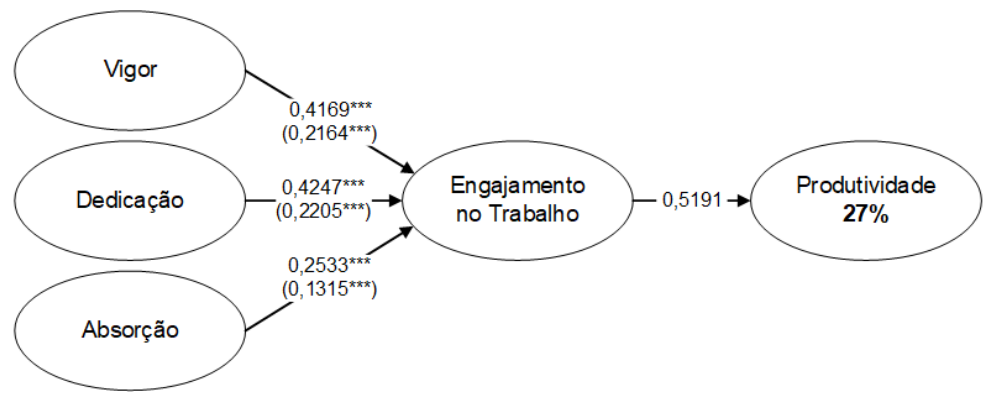

Nota: NS = não significante. ${ }^{* * *} \mathrm{p}<0,01 . * * \mathrm{p}<0,05 . * \mathrm{p}<0,10 . \mathrm{O}$ valor entre parênteses refere-se ao efeito total do respectivo construto no construto produtividade.

Fonte: Elaborada pela autora.

A síntese dos resultados para o grupo homem é apresentada na figura 2.

Figura 2 - Síntese dos Resultados (Grupo Homem)

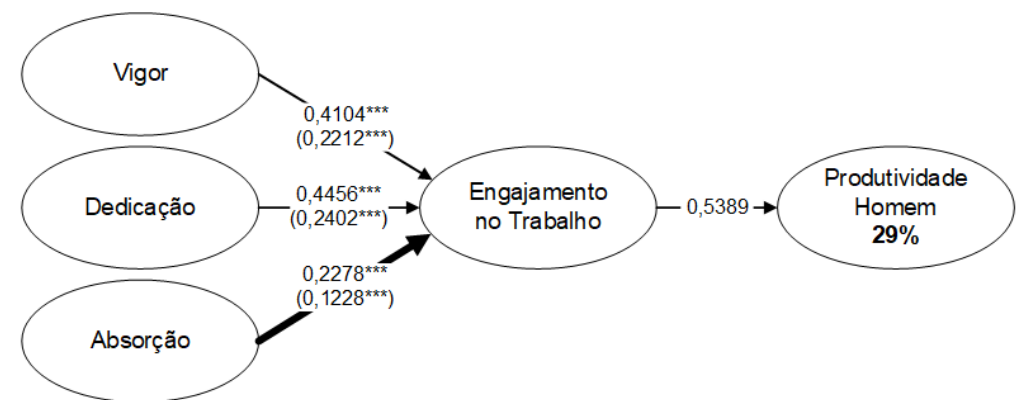

Nota: NS $=$ não significante. $* * * p<0,01 . * * \mathrm{p}<0,05 . * \mathrm{p}<0,10 . \mathrm{O}$ valor entre parênteses refere-se ao efeito total do respectivo construto no construto produtividade.

Fonte: Elaborada pela autora.

A síntese dos resultados para o grupo mulher é apresentada na Figura 3 - Síntese dos Resultados (Grupo Mulher)

3.

Figura 3 - Síntese dos Resultados (Grupo Mulher) 


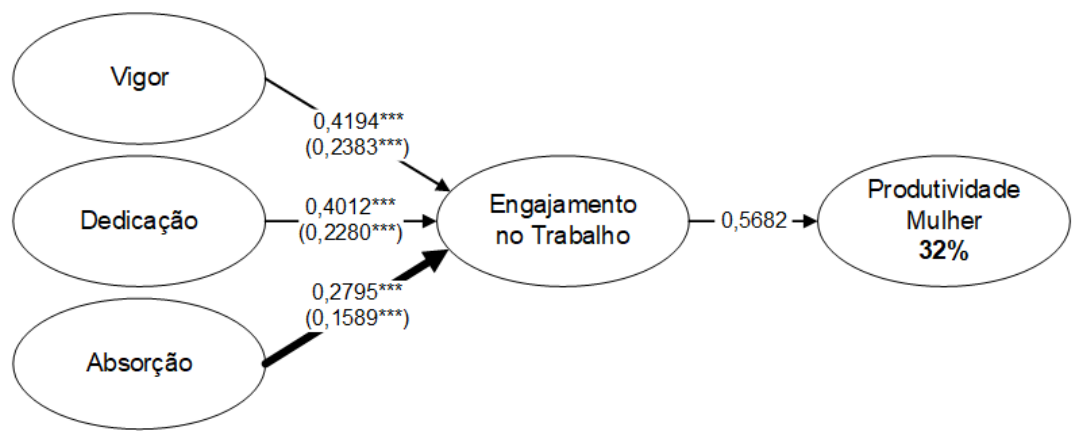

Nota: NS = não significante. $* * * \mathrm{p}<0,01 . * * \mathrm{p}<0,05 . * \mathrm{p}<0,10 . \mathrm{O}$ valor entre parênteses refere-se ao efeito total do respectivo construto no construto produtividade.

Fonte: Elaborada pela autora.

A base de questionamento, que constitui a presente pesquisa, está na análise das relações existentes entre o engajamento no trabalho e a produtividade. A partir dos dados, é possível perceber que os construtos possuem relações, pois esses dados mostraram que o engajamento no trabalho, com suas dimensões, é capaz de explicar a variação de aproximadamente $27 \%$ da produtividade do trabalhador estudado.

O engajamento no trabalho, construto representado por um estado mental caracterizado por dimensões como o vigor, a dedicação e a absorção (BAKKER; DEMEROUTI, 2007; BAKKER et al., 2008; SCHAUFELI et al., 2002) se trata de uma atitude, que o funcionário possui, de melhorias em seu trabalho e, consequentemente, de melhorias em prol da eficácia organizacional (ROBINSON et al., 2004). O engajamento no trabalho impacta a produtividade, construto que, nesta pesquisa, é conceituado a partir da área da psicologia, e está relacionado a questões comportamentais advindas do trabalhador (CUNHA, 2003). Ou seja, o estudo das capacidades psicológicas que influenciam o desempenho organizacional, sintetizado na ideia da produtividade da organização, que visa à eficácia a partir do aproveitamento de seus recursos humanos.

Identifica-se que há uma correlação entre essa produtividade e o engajamento dos trabalhadores. O engajamento no trabalho reflete o compromisso e envolvimento de um colaborador, visando à melhoria do desempenho organizacional (SUNDARAY, 2011). Para Taiwo (2010), a produtividade da organização implica em atingir, a partir do menor dispêndio de recursos, o mais alto nível de desempenho.

O construto engajamento no trabalho é caracterizado por três dimensões (o vigor, a dedicação e a absorção). É um construto motivacional positivo, que implica sentimentos de realização, relacionados ao próprio trabalho, que possuem permanência no tempo e apresentam, assim, uma natureza motivacional e social (BAKKER; DEMEROUTI, 2007; BAKKER et al., 2008; LLORENS et al., 2007; SALANOVA et al., 2000; SALANOVA; SCHAUFELI, 2009; SCHAUFELI et al., 2002). Os dados desta pesquisa mostraram, tal como os autores citados, a relação dessas dimensões, que caracterizam o engajamento no trabalho.

Os resultados obtidos nesta pesquisa comprovaram a relação proposta em uma das hipóteses suportadas: de que quanto maior o vigor o maior a produtividade do trabalhador e, ainda, de que o engajamento no trabalho está relacionado a um estado mental caracterizado pelo vigor, uma das três dimensões (BAKKER; DEMEROUTI, 2007; BAKKER et al., 2008; LLORENS et al., 2007; SALANOVA et al., 2000; SALANOVA; SCHAUFELI, 2009; SCHAUFELI et al., 2002). O vigor é caracterizado por um efeito positivo, em que os trabalhadores experimentam características de altos níveis de energia e resiliência mental enquanto

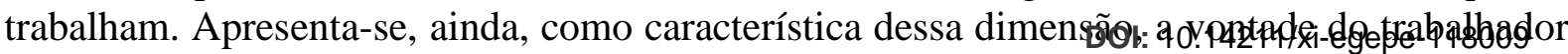


ROMÁ et al., 2006; HAHM, 2017; KATARIA et al., 2012; SALANOVA et al., 2000; SCHAUFELI, 2002).

Portanto, além de o vigor formar uma das dimensões do engajamento no trabalho, conforme os autores mencionam, e como se comprova nesta pesquisa, essa dimensão também impacta na produtividade do trabalhador.

Com esta pesquisa, é possível comprovar ainda, sobre o vigor, que a produtividade da organização advém de fatores relacionados a ele, que, como afirmam Kataria et al. (2012), é um elemento crítico para o sucesso dos negócios. O vigor advém do engajamento, sentido pelos trabalhadores como uma experiência prazerosa, que impulsiona a proatividade.

Os resultados desta pesquisa comprovam que a produtividade do trabalhador será maior quanto maior a dimensão dedicação. Essa dimensão está relacionada a uma estrutura de cunho emocional, reveladora do grau de envolvimento e concentração desse trabalhador com seu trabalho, que encontra significado nele (ACOSTA; SALANOVA; LLORENS, 2013; BAKKER; LEITER, 2010; BAKKER et al., 2012; GONZÁLEZ-ROMÁ et al., 2006; SALANOVA et al., 2000; SCHAUFELI, 2009; YONGXING et al., 2017). Portanto, essa dimensão, se caracteriza como um dos componentes do engajamento no trabalho, conforme os autores citados, como se comprovou também nesta pesquisa. É uma dimensão característica desse construto: quanto maior a dedicação no trabalho, maior é a produtividade do trabalhador.

Assim sendo, identifica-se que trabalhadores engajados estão envolvidos e se dedicam, de forma mais concentrada e intensa, em suas atividades (BAKKER et al., 2010, 2011). E, consequentemente, essa dedicação, presente em trabalhadores engajados, traz reflexos positivos para as organizações, em termos de alto comprometimento, proficiência de tarefas, diminuição do absenteísmo e da rotatividade de pessoas, aumento da produtividade, e, consequentemente, aumento no desempenho da organização (BHATNAGAR, 2012). Logo, é possível perceber que a dimensão dedicação impacta a produtividade do trabalhador, podendo, consequentemente, justificar o aumento no desempenho da organização estudada nos últimos anos.

A dimensão de aspecto cognitivo explicita o que os trabalhadores experimentam quando envolvidos com o trabalho, de forma que sentem dificuldades de separar-se das atividades do trabalho de tão concentrados que se encontram (BAKKER et al., 2008; GONZÁLEZ-ROMÁ, 2006; KATARIA; GARG; RASTOGI, 2012; SCHAUFELI et al., 2002; SALANOVA; SCHAUFELI, 2009; XANTHOPPOULOU, 2009). Essa dimensão compõe o engajamento no trabalho, como identificado também nesta pesquisa. Ainda de acordo com os dados, essa é uma dimensão que impacta a produtividade do trabalhador. Nessa dimensão, é ainda perceptível uma diferença estatisticamente significante entre os grupos de homens e mulheres pesquisados: as mulheres se percebem mais absortas no trabalho do que os homens. Esta percepção implica em maior percentual de produtividade explicada pelo engajamento.

É possível ainda, de acordo com Truss et al. (2003), com apoio nos resultados desta pesquisa, identificar que as mulheres se encontram mais focadas e mais ligadas a suas tarefas de trabalho, sentindo que o tempo passa mais rapidamente do que para os homens. Além disso, encontram ainda mais dificuldade em se separar do trabalho do que os homens.

A produtividade, de acordo com Sharma e Sharma (2014), está relacionada à quantidade de tempo em que um funcionário está fisicamente presente seu trabalho, além do grau em que ele está "mentalmente presente" ou trabalhando eficientemente durante o horário de trabalho. Sendo assim, ao medir a dimensão absorção, percebendo-a, como já mencionado por Xanthopoulou (2009), como um sentimento de dificuldades que o trabalhador experimenta ao ter que se separar das atividades do trabalho, por estar tão concentrado e envolvido, é possível inferir que as mulheres são mais produtivas que os homens.

DOI: 10.14211/xi-egepe-118009 
Em relação à produtividade, então, é perceptível, no embasamento teórico desta pesquisa, o quanto esse construto representa uma das variáveis economicamente mais importantes (SINGH; MOTWANI; KUMAR, 2000), o quanto contém de fatores que influenciam a produtividade da organização e quão importante se torna, como ferramenta, para manter essa organização em vantagem competitiva (GROSSMAN, 1993). Sendo assim, como as relações se mostraram positivas e estatisticamente significantes, indica-se que as hipóteses foram suportadas. Ou seja, quanto maior o engajamento no trabalho, quanto maior o vigor no trabalho, quanto maior a dedicação no trabalho e quanto maior a absorção no trabalho, maior é a produtividade do trabalhador.

\section{CONCLUSÃO}

Esta pesquisa objetivou analisar as relações existentes entre engajamento no trabalho e produtividade, mediante a utilização de uma pesquisa quantitativa, que se baseou em registros e em análise de dados numéricos, no que diz respeito às atitudes e aos comportamentos dos trabalhadores quanto aos dois temas. A indagação foi plenamente respondida.

Procedeu-se, inicialmente, ao levantamento dos conceitos e ideias que originaram o construto engajamento no trabalho - com suas dimensões: vigor, dedicação e absorção - e suas relações com a produtividade.

Identificou-se a necessidade de tratar o tema, uma vez que muito tem se falado a respeito de engajamento no trabalho, atribuindo a ele fatores estritamente motivacionais, principalmente, mas sem sequer mencionar quais as dimensões o compõem e sem sequer avaliar seus impactos na produtividade do trabalhador. Acrescido a esse fator, está o crescimento da organização, alvo desta pesquisa, que nos últimos três anos vem apresentando crescimento físico, econômico e de mercado, que tornam plausível este estudo.

Evidenciou-se que a produtividade possui relação com o engajamento no trabalho e, ainda, que a intensidade do engajamento no trabalho impacta na produtividade do trabalhador. E também que os fatores que constituem o engajamento no trabalho (o vigor, a dedicação e a absorção) influenciam na produtividade do trabalhador. Dessa forma, esta pesquisa cumpre os objetivos específicos de identificar a intensidade do engajamento na produtividade e verificar suas influências e seus impactos na produtividade.

Os resultados obtidos nesta pesquisa comprovaram o aumento da produtividade, na organização estudada, como advindo do engajamento de seus trabalhadores. Foram confirmadas as seguintes hipóteses: quanto maior o engajamento no trabalho, maior a produtividade do trabalhador; quanto maior o vigor no trabalho, maior a produtividade do trabalhador; quanto maior a dedicação no trabalho, maior a produtividade do trabalhador e, quanto maior a absorção no trabalho, maior a produtividade do trabalhador.

Evidenciou-se que muito tem se estudado a respeito do termo produtividade ao longo dos anos. A respeito de engajamento no trabalho, tem-se estudado menos, ainda que seja objeto de estudo de inúmeros autores. Portanto, no Brasil, esta é uma pesquisa inédita, que faz a correlação entre os dois construtos e comprova a relação entre eles. Seu público de pesquisa de engajamento no trabalho é composto por trabalhadores de diversos outros segmentos, tais como a indústria, o transporte, a educação, dentre outros. Porém, com trabalhadores de empresa de pequeno porte no varejo, esta pesquisa é pioneira, o que evidencia sua contribuição e sua inovação para o ambiente acadêmico e profissional.

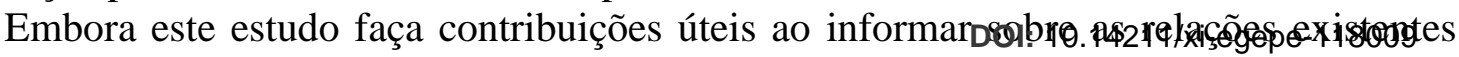


mo fator limitador, a falta de um levantamento de dados que pudessem explicar as razões que causam o engajamento dos trabalhadores que constituem o público desta pesquisa. Buscar compreender quais antecedentes estariam vinculados a esse engajamento no trabalho seria significativo.

Para maior aprofundamento dos temas e estudos futuros, sugere-se a realização de novos estudos, principalmente no Brasil, correlacionando esses temas. E, ainda, mais pesquisas nos diversos setores da economia, a fim de ser possível o estabelecimento de novos dados.

\section{REFERÊNCIAS}

ACOSTA, H.; SALANOVA, M.; LLORENS, S. ¿Cómo predicen las prácticas organizacionales el engagement en el trabajo en equipo? : el rol de la confianza organizacional. Ciencia y Trabajo, [s. l.], v. 13, n. 41, p. 125-134, jul./set. 2013.

BAILY, Martin N.; FARRELL, Diana; GREENBERG, Ezra; HENRICH, Jan-Dirk; JINJO, Naoko; JOLLES, Maya; REMES, Jaana. Increasing global competition and labor productivity: Lessons from the US automotive industry. McKensie Global Institute, [S. l.], v. 7, p. 1146, Nov. 2005.

BAKKER, A. B.; DEMEROUTI, E.; HAKANEN J. J.; XANTHOPOULOU D. Job Resources Boost Work Engagement; particularly when job demands are high. Journal of educational Psychology, Washington, v. 99, p. 274-284, 2007.

BAKKER, Arnold B.; ALBRECHT, Simon L.; LEITER, Michael P. Key questions regarding work engagement. European Journal of Work and Organizational Psychology, [s. l.], v. 20, n. 1, p. 4-28, 2011. https://doi.org/10.1080/1359432X.2010.485352

BAKKER, Arnold B.; DEMEROUTI, Evangelia. The job demands-resources model: State of the art. Journal of Managerial Psychology, [s. l.], v. 22, n. 3, p. 309-328, Apr. 2007. DOI 10.1108/02683940710733115

BAKKER, Arnold B.; LEITER, M. P. Where to go from here: integration and future research on work engagement. In: BAKKER, Arnold B.; LEITER, M. P. (org.). Work engagement: a handbook of essential theory and research. New York: Psychology Press, 2010. p.181-196. BAKKER, Arnold B.; SHAUFELI, Wilmar B.; LEITER, Michel P.; TARIS, Toon W. Work engagement: an emerging concept in occupational health psychology. Work \& Stress - An International Journal of Work Health \& Organizations, [s. l.], v. 22, n. 3, 187-200, Sept. 2008.

BHATNAGAR, Jyotsna. Management of innovation: role of psychological empowerment work engagement and turnover intention in the Indian context. The International Journal of Human Resource Management, [S. 1.], v. 23, n. 5, p. 928-951, 2012.

https://doi.org/10.1080/09585192.2012.651313

CUNHA, M. P. e; CUNHA, R. C. e; REGO, Armênio; NEVES, Pedro; CABRAL-

CARDOSO, C. Manual de Comportamento Organizacional e Gestão. Lisboa: RH Editora, 2003.

DALFOVO, Michael Samir; LANA, Rogério Adilson; SILVEIRA, Amélia. Métodos quantitativos e qualitativos: um resgate teórico. Revista Interdisciplinar Científica Aplicada, $[s$. l.], v. 2, n. 3, p. 1-13, 2008.

FLEMING, John H.; ASPLUND, Jim. Human sigma: Managing the employee-customer 
GONZÁLEZ-ROMÁ, V.; SCHAUFELI, W. B.; BAKKER, A. B.; LLORET, S. Burnout and work engagement: independent factors or opposite poles? Journal of Vocational Behavior, [s. l.], v. 68, p. 165-174, 2006.

GROSSMAN, E. How to Measure Company Productivity: Handbook for Productivity Measurement and Improvement. Cambridge, MA: Productivity Press, 1993.

GUMMESSON, Evert. Productivity, quality and relationship marketing in service operations. In: Handbuch Dienstleistungsmanagement. Wiesbaden: Gabler Verlag, 1998. p. 843-864. HAIR JR., Joseph F.; HULT, G. T. M.; RINGLE, Christian M.; SARSTEDT, Marko. A primer on partial least squares structural equation modeling (PLS-SEM). 2. ed. Thousand Oaks: Sage, 2017.

KATARIA, Aakanksha; GARG, Pooja; RASTOGI, Renu. Employee Engagement and Organizational Effectiveness: The Role of Organizational Citizenship Behavior. International Journal of Business Insights \& Transformation, [s. l.], v. 6, n. 1, Oct. 2012. KOYUNCU, Mustafa; BURKE, Ronald J.; FIKSENBAUM, Lisa. Work engagement among women managers and professionals in a Turkish bank: Potential antecedents and consequences. Equal Opportunities International, [s. l.], v. 25, n. 4, p. 299-310, 2006. LITWIN, Mark S.; FINK, Arlene. How to measure survey reliability and validity. Thousand Oaks, CA: Sage, 1995. DOI: https://dx.doi.org/10.4135/9781483348957

LLORENS, S.; SCHAUFELI, W.; BAKKER, A.; SALANOVA, M. Does a positive gain spiral of resources efficacy beliefs and engagement exist? Computers in Human Behavior, [s. 1.], v. 23, p. 825-841, 2007.

MARTINS, P. G.; LAUGENI, F. P. Administração da produção. 2. ed. São Paulo: Saraiva 2006.

MINAYO, M. C. Pesquisa Social: teoria método e criatividade. Petrópolis: Vozes, 2002. OBDULIO, D. L. How management can improve corporate culture in order to have an effective work environment. Trade Publication, [s. l.], v. 75, n. 8, p. 14, 2014.

PRITCHARD, R. D. Organizational productivity. In: DUNNETTE, M. D.; HOUGH, L. M. (ed.). Handbook of Industrial and Organizational Psychology. Palo Alto, CA: Consulting Psychologists Press, 1992. v. 3, p. 443-472.

RAUPP, Fabiano Maury. Metodologia da pesquisa aplicável às ciências sociais. In: BEUREN, Ilse Maria (org.). Como elaborar trabalhos monográficos em contabilidade: teoria e prática. 3. ed. São Paulo: Atlas, 2006. p. 76-97.

ROBINSON, Dilys; PERRYMAN, Sarah; HAYDAY, Sue. The drivers of employee engagement. Brighton, UK: Institute for Employment Studies, 2004.

SALANOVA, M.; SCHAUFELI, W. B.; LLORENS, S; PEIRO, J. M; GRAU, R. Desde el "burnout" al "engagement": ¿una nueva perspectiva? Revista de Psicología del Trabajo y de las Organizaciones, [s. l.], v. 16, n. 2, p. 117-134, 2000.

SCHAUFELI, W. B.; SALANOVA Marisa; GONZÁLEZ-ROMÁ, Vicente; BAKKER, Arnold $\mathrm{B}$. The measurement of engagement and burnout: a two-sample confirmatory factor analytic approach. Journal of Happiness Studies, [S. l.], v. 3, p. 71-92, 2002.

SCHAUFELI, Wilmar B. Work engagement and financial returns: A diary study on the role of job and personal resources. Journal of Occupational and Organizational Psychology, [S. l.], v. 82, n. 1, p. 183-200, 2009.

SHARMA, M. S.; SHARMA, M. V. Employee engagement to enhance productivity in current scenario. International Journal of Commerce Business and Management, [s. l.], v. 3, n. 4, p. 595-604, 2014.

SHIROM, Arie. Feeling vigorous at work? The construct of vigor and the study of 118009 
(Ed.). Emotional and Physiological Processes and Positive Intervention Strategies.

[S. 1.]: Emerald Group Publishing Limited, 2003. p. 135-164. (Research in Occupational Stress and Well-being, Volume 3).

SHUCK, Brad; WOLLARD, Karen. Employee engagement and HRD: A seminal review of the foundations. Human Resource Development Review, [s. l.], v. 9, n. 1, p. 89-110, 2010. SINGH, Harinder; MOTWANI, Jaideep; KUMAR, Ashok. A review and analysis of the stateof-the-art research on productivity measurement. Industrial Management \& Data Systems, [s. l.], v. 100, n. 5, p. 234-241, 2000.

SUNDARAY, Bijaya Kumar. Employee engagement: A driver of organizational effectiveness. European Journal of Business and Management, [s. l.], v. 3, n. 8, p. 53-59, 2011. TRUSS, Catherine ; SHANTZ, Amanda ; SOANE, Emma ; ALFES, Kerstin; DELBRIDGE, Rick. Employee engagement, organisational performance and individual well-being: exploring the evidence, developing the theory. The International Journal of Human Resource Management, [s. l.], v. 24, n. 14, p. 2657-2669, 2013.

WELCH, Mary. The evolution of the employee engagement concept: communication implications. Corporate Communications: An International Journal, [s. l.], v. 16, n. 4, p. 328346, 2011.

WOOD JR., Thomaz; CALDAS, Miguel P. Empresas brasileiras e o desafio da competitividade. RAE - Revista de Administração de Empresas, São Paulo, v. 47, n. 3, p.1-13, set. 2007.

XANTHOPOULOU, Despoina; BAKKER, Arnold B.; DEMEROUTI, Evangelia; SCHAUFELI, Wilmar B. Work engagement and financial returns: A diary study on the role of job and personal resources. Journal of Occupational and Organizational Psychology, [s. l.], v. 82, n. 1, p. 183-200, 2009.

YONGXING, Guo; HONGFEI, Du; BAOGUO, Xie; LEI, Mo. Work engagement and job performance: the moderating role of perceived organizational support. Anales de Psicología/Annals of Psychology, $\left[\begin{array}{lllllllll}S . & l .], & \text { v. } & 33, & \text { n. } & 3, & \text { p. } & 708-713, & 2017 .\end{array}\right.$ DOI: https://doi.org/10.6018/analesps.33.3.238571 